Forward and Inverse Problems in Towed Cable Hydrodynamics

Polydorides, Nick and Storteig, Eskild and Lionheart, William

2008

MIMS EPrint: 2008.73

Manchester Institute for Mathematical Sciences

School of Mathematics

The University of Manchester

\footnotetext{
Reports available from: http://eprints.maths.manchester.ac.uk/

And by contacting: The MIMS Secretary

School of Mathematics

The University of Manchester

Manchester, M13 9PL, UK
} 


\title{
FORWARD AND INVERSE PROBLEMS IN TOWED CABLE HYDRODYNAMICS
}

\author{
N. POLYDORIDES†, E. STORTEIG $\ddagger$ W. LIONHEART§
}

\begin{abstract}
This paper addresses the problem of reconstructing the velocities of the ocean currents impinging on a towed streamer cable during an offshore seismic survey. This study considers a two-dimensional model describing the motion of a flexible, inextensible cable in the presence of hydrodynamic drag forces in an incompressible fluid. In the first part the forward model is introduced and then solved to yield the cable's velocity, curvature and tension in the knowledge of the towing vessel motion and the hydrodynamic loads applied. In sequence, we formulate the inverse problem of inferring the ocean current velocities from discrete samples of the cable's shape and tension and show that this is rank deficient and ill-posed. In approaching the inverse problem a numerically stable algorithm is adopted based on generalized Tikhonov regularization, in the context of robust differentiation of discrete noisy signals. In order to demonstrate the practical performance of the scheme, some examples of ocean current reconstructions obtained using simulated noisy data are presented.
\end{abstract}

\section{INTRODUCTION}

The aim of this work is to derive a robust methodology of inferring the velocities of ocean currents as these are important for optimizing the controls of streamer cables in towed array seismic imaging (Storteig et al. 2007). Accurate positioning or repositioning of the acoustic sensors attached to the towed cable has become important since the seismic market to a large extent has moved from exploration of new areas to reservoir monitoring over the last decade. In a recent survey (Nakstad et al. 2008) it has been reported that recovery rate from oil reservoirs is currently of the order of $50 \%$ before these are abandoned and that reservoir monitoring is now essential to enhance this rate. In this context, a series of seismic surveys are performed repeatedly to make plans for proper reservoir depletion. A key factor for successfully imaging a reservoir change is to reposition the seismic source and acoustic receivers accurately. A desirable target for repositioning is in the order of one meter of positioning error although admittedly this is still far from being met. Several technological improvements in detecting and thereafter controlling the positioning of the cables are required to reach such a target. Variable ocean currents make the task of controlling hydrophone positions a major challenge for the seismic industry. Steerable deflectors spaced along streamer cables several kilometers long is one of the methods used to counteract the currents. However, elegant steering is essential in order to avoid cross flow and strumming which are detrimental to the noise levels on the hydrophones. As the local variations of current along the streamer are not known the control algorithms used to steer the deflectors are based on a feedback approach. This implies that the initial survey will, in general, not follow an optimal predefined trajectory, and making the repeatability challenge even 
greater. The approach can be improved substantially with a feed forward algorithm based on the knowledge of the approaching ocean current. Moreover, streamer control becomes also imperative in three-dimensional seismic surveys employing multiple streamer configurations. In such a case rigorous real time steering control becomes critical as variable sea currents can cause the cables to tangle essentially bringing the survey to an abrupt end.

The common practice in offshore seismic surveys is to measure the real time current at the vessel and scattered buoys only. The value of this information is limited since the current may vary considerably in time and space. In this framework, the necessity for a technique to recover in real time the ocean current velocity profiles (OCVP) acting on the cable is profound. The ultimate goal of such a method will be to monitor the ocean currents in real time so that to optimize the steering of the towed cables and hence improve the quality of the hydrophone data. In this work we address this problem in the context of an inverse parameter identification problem, where one seeks to recover the OCVP along the streamer using positioning and tension measurements. Positioning data are essentially samples of the cable's velocity and shape as these are acquired via a GPS sensor network. More precisely, the inverse problem is to find the ocean current field distribution along the cable that yields the observable cable shape and tension.

To setup the framework of our methodology we incorporate a model of a flexible, inextensible cable of finite length and uniform circular diameter, towed by a survey vessel moving at a nearly constant speed on a smooth trajectory. For the application at hand, the streamer cable is assumed to be neutrally buoyant with its velocity components parallel to the sea surface dominating the transverse. For this reason a simplified two dimensional model of motion is considered. The spatial and temporal resolution specifications of the model are set in accordance to the realistic conditions of an offshore survey. The spatial discretization of the cable should conform with the positioning of GPS transmitters and tension meters, while the increment in the temporal dimension should reflect the rate at which real measurements are collected. For this study the objective is to demonstrate that the proposed inverse method is suitable for real time reconstruction of OCVP. The technique is described for a single cable configuration although its generalization to multiple streamers is trivial subject to the availability of computational resources.

The paper is organized as follows: in the next section we present the forward model that describes the motion of the cable in the influence of hydrodynamic forces and then briefly discuss its numerical solution using a finite difference approximation scheme. In sequence, we derive the inverse problem by reformulating the forward model in a robust integral formulation before attending to its solution using generalized Tikhonov regularization. The numerical characteristics of the inverse problem are outlined in the context of a singular value analysis and sensitivity discussion based on which we also address its robustness against noise and uncertainties in the drag coefficients involved. Finally, a few numerical examples are presented to demonstrate the performance of the method with simulated data.

\section{FORWARD HYDRODYNAMIC MODEL}

Assuming negligible inertia terms at the range of towing velocities, Newton's law of motion for a towed cable is expressed in terms of the momentum equations in a 
tangential-normal coordinate frame

$$
\begin{gathered}
\frac{\partial T}{\partial s}-\frac{1}{2} \rho \pi d C_{t} V_{t r}\left|V_{t r}\right|=0 \\
T \frac{\partial \theta}{\partial s}-\frac{1}{2} \rho d C_{n} V_{n r}\left|V_{n r}\right|=0
\end{gathered}
$$

describing the distribution of forces along the cable immersed in an incompressible fluid (Dowling 1988). For the same system the kinematic relations

$$
\begin{gathered}
\frac{\partial V_{n}}{\partial s}+V_{t} \frac{\partial \theta}{\partial s}-\frac{\partial \theta}{\partial t}=0 \\
\frac{\partial V_{t}}{\partial s}-V_{n} \frac{\partial \theta}{\partial s}=0
\end{gathered}
$$

indicate the influence of the cable's shape and orientation to the corresponding velocity components. In the above equations $T(s, t)$ denotes the tension at distance $s$ from the tow-point at time $t, \rho$ is the density of sea water, $d$ the diameter of the cable, $C_{t}$ is the tangential drag coefficient, $C_{n}$ the normal drag coefficient, $\theta(s, t)$ denotes the orientation angle between the tangent vector on $s$ and the positive $x$ Cartesian axis, and its gradient $\partial \theta / \partial s$ is the curvature of the cable. The cable's relative velocities in the tangential and normal directions, $V_{t r}(s, t)$ and $V_{n r}(s, t)$ respectively, are expressed with respect to the corresponding ocean currents $u(s, t)$ and $v(s, t)$

$$
V_{t r}=V_{t}-u, \quad V_{n r}=V_{n}-v
$$

where $V_{t}(s, t)$ and $V_{n}(s, t)$ are the absolute cable velocities at the same axes. The parabolic system (1) - (4) refers to a two-dimensional frame $(\mathbf{t}, \mathbf{n})$ obtained from the cartesian $(\mathbf{x}, \mathbf{y})$ through an orthogonal rotation by an angle $\theta$. Here $\mathbf{t}(s)$ denotes the unit tangent vector at the point $s$ on the cable, and $\mathbf{n}(s)$ the unit normal. A typical sketch of the configuration of the towing system appears in figure 1.

If initially the system is considered to be at rest, four boundary conditions are physically imposed to the system. As the streamer is hinged at the towing vessel, the velocity of the front end of the streamer will be

$$
\begin{gathered}
V_{t}(0, t)=\nu_{1}(t) \cos \theta(0, t)+\nu_{2}(t) \sin \theta(0, t) \\
V_{n}(0, t)=-\nu_{1}(t) \sin \theta(0, t)+\nu_{2}(t) \cos \theta(0, t)
\end{gathered}
$$

where $\theta(0, t)$ is the orientation angle at the beginning of the cable, and $\nu_{1}(t)$ and $\nu_{2}(t)$ are the cartesian components of the vessel's speed at time $t$, here set to $|\boldsymbol{\nu}|=2.57 \mathrm{~m} / \mathrm{s}$ or 5 knots. For the conditions (6)-(7) notice that these provide boundary information about the velocities of the cable in a parametric form requiring a priori knowledge of the angle at the tow-point in order to specify the velocities there. Gatti in (Gatti 2002) quotes that this is a major source of instabilities observed in numerical implementations of the model even with unconditionally stable approximation methods, although the problem can be alleviated by enforcing smooth vessel manoeuvering and fine temporal discretization.

In the reports (Gobat et al. 2001), (Hover et al. 1994), and (Milinazzo et al. 1987), the authors consider the tension at the tail to be zero as the end is free and thus the sum of forces and moments there theoretically vanish. However, at the end of a seismic cable it is customarily equipped with a surface GPS buoy that contributes a significant drag force (Hui 2005). In fact this maintains a tail tension, 


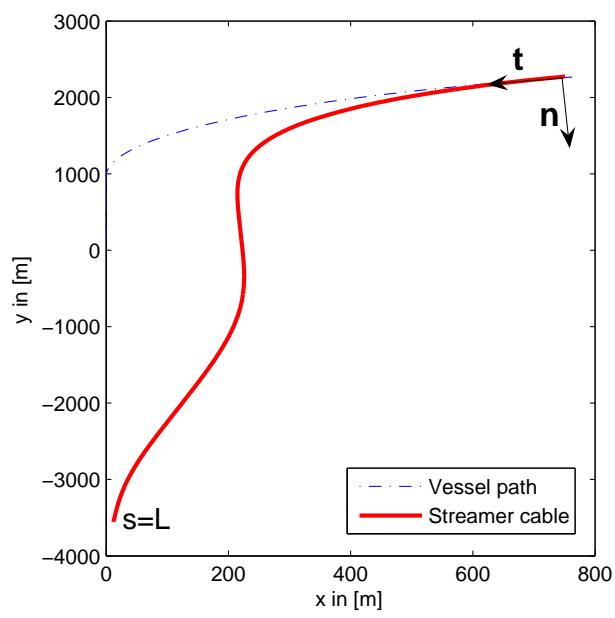

FiguRE 1. The streamer cable in the local and global coordinate systems in $2 \mathrm{D}$. Notice the direction of $\mathbf{t}$ vector facing at the increasing order of $s$.

essentially a lower bound for the tension profile, that scales linearly to the norm of the cable's velocity there

$$
T(L, t)=2.57^{-1} T_{L}\left(V_{t}^{2}(L, t)+V_{n}^{2}(L, t)\right)^{1 / 2}
$$

assuming a correspondence of $T_{L}=2000 \mathrm{~N}$ at a speed of $2.57 \mathrm{~m} / \mathrm{s}$. Moreover, the tension regulating buoy maintains the tail of the cable straight thus

$$
\frac{\partial \theta(L, t)}{\partial s}=0 \quad \Leftrightarrow \quad V_{n}(L, t)=v(L, t)
$$

which along with (8) and (2) deduce that the normal velocity of the cable at the tail end is merely due to the cross-line currents. Further to the above conditions, in the numerical implementation of the model we assume the cable material to be homogeneous and isotropic and elastic with circular cross section $d$. Moreover we assume the cable to be inextensible with negligibly small shear deformation and momentum terms.

\section{NUMERICAL SOLUTION OF FORWARD PROBLEM}

In order to derive the numerical solution for the system (1)-(4) and (6)-(9) the problem is transferred from the continuous domain to the discrete $[0, L] \times\left[0, t_{f}\right]$ using the finite difference time domain method (FD). In particular, the space-time problem is first transformed into a spatial two-point boundary value problem using finite differences in time and space and then the resulting nonlinear differential equations are approximated by the first order Taylor series expansion to obtain a linear two point boundary value problem. The resulted system of algebraic equations is subsequently solved using Newton's method (Vogel 2002). For the scope of this work an implicit FD scheme is adopted based on the generalized trapezoidal rule (Hughes 1987). In particular, the first space derivative of $\mathbf{z}$ is approximated 
by

$$
\frac{\partial \mathbf{z}}{\partial s}(s, t)=\frac{\mathbf{z}(s, t)-\mathbf{z}(s-\Delta s, t)}{a \Delta s}-\gamma \frac{\partial \mathbf{z}}{\partial s}(s-\Delta s, t)
$$

where the parameters $a$ and $\gamma$ satisfy $a(\gamma+1)=1$. The method is proved to be stable for $a$ and $\gamma$ in certain closed intervals, while the special case for $\gamma=0$ is the unconditionally stable backward finite difference method. More details on the selection of these parameters and the trade off between accuracy of approximation and stability can be found in (Gatti 2002). Similar numerical schemes like the box method obtain similar performance in terms of the accuracy of the approximation but are reportedly more prone to numerical instabilities. The generalized alpha method suggested in (Gobat 2000) and (Gobat et al. 2001) rectifies the instability by enforcing additional temporal averaging on the conventional box method scheme of (, Ablow et al. 1983). In applying the derivative discretization rule (10) the forward problem in consideration is cast in a matrix form as (Milinazzo et al. 1987),

$$
\mathbf{N} \dot{\mathbf{z}}+\mathbf{M} \mathbf{z}^{\prime}+\mathbf{Q}=0
$$

where

$\mathbf{N}^{i+1}=\left(\begin{array}{cccc}0 & 0 & 0 & 0 \\ 0 & 0 & 0 & 0 \\ 0 & 0 & 0 & -1 \\ 0 & 0 & 0 & 0\end{array}\right), \mathbf{M}^{i+1}=\left(\begin{array}{cccc}1 & 0 & 0 & 0 \\ 0 & 1 & 0 & -V_{n} \\ 0 & 0 & 1 & V_{t} \\ 0 & 0 & 0 & T\end{array}\right), \mathbf{Q}^{i+1}=\left(\begin{array}{c}-\frac{1}{2} \rho \pi d C_{t} V_{t r}\left|V_{t r}\right| \\ 0 \\ 0 \\ -\frac{1}{2} \rho d C_{n} V_{n r}\left|V_{n r}\right|\end{array}\right)$

and $\mathbf{z}=\left(T, V_{t}, V_{n}, \theta\right)$. In the notation the superscript $i$ denotes the time index while the subscript $j$ denotes the space index. Here we consider discrete time instances $i$ where the equation holds, separated by uniform time increments $\Delta t$ so that $i=k \Delta t$ for $k=0,1, \ldots$ and $i \in\left[0, t_{f}\right]$ for $t_{f}$ the time the simulation ends. The problem is also discretized in the one dimensional space domain by considering an array of $n$ interconnected nodes separated by a distance $\Delta s$. In this arrangement the $j$ 'th node is situated at a distance $j=k \Delta s$ from the front end of the cable with $k=0, \ldots, n-1$ and $j \in[0, L]$. Although of comparable measure, space and time discretization need not necessarily be uniform, however taking constant increments simplifies the computational complexity.

The assembling of the coefficients matrices in (11) follows in the pattern of the conventional finite element scheme where these are iteratively populated by working out the contributions of the local elements. Consequently, for a model with $n$ nodes the FD procedure yields at each time step $i, 4(n-1)$ equations in $4 n$ unknowns $\mathbf{z}$ while the remaining four equations required to preserve uniqueness in the solution of (11) arise by discretizing the boundary conditions (6)-(9) in a similar manner. Eventually the FD scheme yields a system of $4 n$ nonlinear algebraic equations

$$
\Phi\left(\mathbf{z}_{j=1: n}^{i}, \mathbf{z}_{j=1: n}^{i+1}\right)=0
$$

which, provided a state solution $\mathbf{z}_{j=1: n}^{i}$ yields the next state $\mathbf{z}_{j=1: n}^{i+1}$ by computing the roots of the resulting nonlinear equations. For example, starting from the knowledge of the initial state $\mathbf{z}^{0}$ at $t=0$, the roots of the nonlinear $\Phi\left(\mathbf{z}_{j=1: n}^{0}, \mathbf{z}_{j=1: n}^{1}\right)=0$ include the desired forward solution $\mathbf{z}^{1}$ at $t=\Delta t$. The nonlinear system (12) is solved using Newton's method (Vogel 2002). Assuming a reasonably small time 
increment $\Delta t$, then subject to some mild assumptions, every pair of consecutive state solutions satisfies

$$
\left\|\mathbf{z}_{j=1: n}^{i}-\mathbf{z}_{j=1: n}^{i+1}\right\|_{2} \leq \tau
$$

where $\tau>0$ a small positive scalar which tends asymptotically to zero as $\Delta t \rightarrow 0$. From the first-order Taylor expansion of $\Phi$, if $\mathbf{z}^{i+1}=\mathbf{z}^{i}+\delta \mathbf{z}$ then

$$
\delta \mathbf{z}=-\left[\frac{\partial}{\partial \mathbf{z}^{i}} \Phi\left(\mathbf{z}_{j=1: n}^{i}, \overline{\mathbf{z}}_{j=1: n}^{i}\right)\right]^{-1} \Phi\left(\mathbf{z}_{j=1: n}^{i}, \overline{\mathbf{z}}_{j=1: n}^{i}\right)
$$

where $\overline{\mathbf{z}}_{j=1: n}^{i}$ denotes the current state of the system to which the boundary conditions for the next state $i+1$ have been imposed. If the boundary condition equations are elegantly indexed within the system (12) the Jacobian $\partial \Phi / \partial \mathbf{z}$ becomes a tridiagonal matrix with immediate impact on the computational efficiency of the forward simulator. Apart from Newton's method, alternative schemes for the forward problem include the Runge-Kutta integration which is implemented on the space dependent differential equations after the time derivatives are eliminated by FD discretization (Ablow et al. 1983). The method is fairly robust to numerical instabilities although special suppression routines like those implemented in (Gatti 2002) and (Gobat 2000) are necessary to ensure that the solution remains a bounded norm in the presence of Crank-Nicholson noise.

The forward simulator was implemented in Matlab (Matlab 2007), considering for a cable of length $L=6000 \mathrm{~m}$ and diameter $d=0.05 \mathrm{~m}$. The cable was towed on a vessel executing a northbound orbit at a constant speed of $|\boldsymbol{\nu}|=2.57 \mathrm{~m} / \mathrm{s}$. The water density $\rho$ was set to $1025 \mathrm{~kg} \mathrm{~m}^{-3}$ and the tangential and normal drag coefficients were assumed fixed at $C_{t}=0.006, C_{n}=2$. To simulate the ocean currents combinations of trigonometric functions were used, varying in amplitude and frequency with space and time. In fixed Cartesian coordinates the ocean velocity profiles normalized by the length of the cable and the duration of the simulation are given by

$$
\left(\begin{array}{l}
c_{x}(x, t) \\
c_{y}(y, t)
\end{array}\right)=\left(\begin{array}{c}
2 t t_{f}^{-1} \sin \left[8 t \sin \left(\frac{\pi(x-1)}{n-1}\right)\right] \\
2 t t_{f}^{-1} \sin \left[8 t\left(\frac{\pi(y-1)}{n-1}\right)\right]
\end{array}\right) \quad x, y=1, \ldots, n, t=1, \ldots, t_{f}
$$

In the implementation of the FD scheme a grid of $n=1201$ nodes equally distributed into $\Delta s=5 \mathrm{~m}$ elements was incorporated, the time step was set to $\Delta t=10 \mathrm{~s}$ and the relaxation parameters in (10) were calibrated at $a=0.9$ and $\gamma=0.1$. The forward system (12) was solved to an error tolerance of $10^{-4}$ yielding data for $0 \leq t \leq 3000 \mathrm{~s}$, some of which are plotted in the graphs of figure 2 along with the corresponding OCVP. The graphs are indicative of the smooth spatial variation of the tension and positioning data under the influence of smooth hydrodynamic excitation. This remark is of great importance for the inverse problem as the choice of the inverse methodology requires a priori knowledge of the differentiability of the forward data. Moreover, as anticipated, tension is a strictly decreasing function that embodies the frequency spectrum components of the inline currents. The tangential cable velocity is maintained smooth and negative given the default orientation of the tangent coordinate vector (c.f. figure 1), while cable's normal velocity profile is also smooth, although it appears to admit a greater variation. The computational times for the forward problem based on a $1.8 \mathrm{GHz}$ Windows XP workstation with 2 GB RAM were recorded at the range of $1 \mathrm{~s}$ per time step execution. 

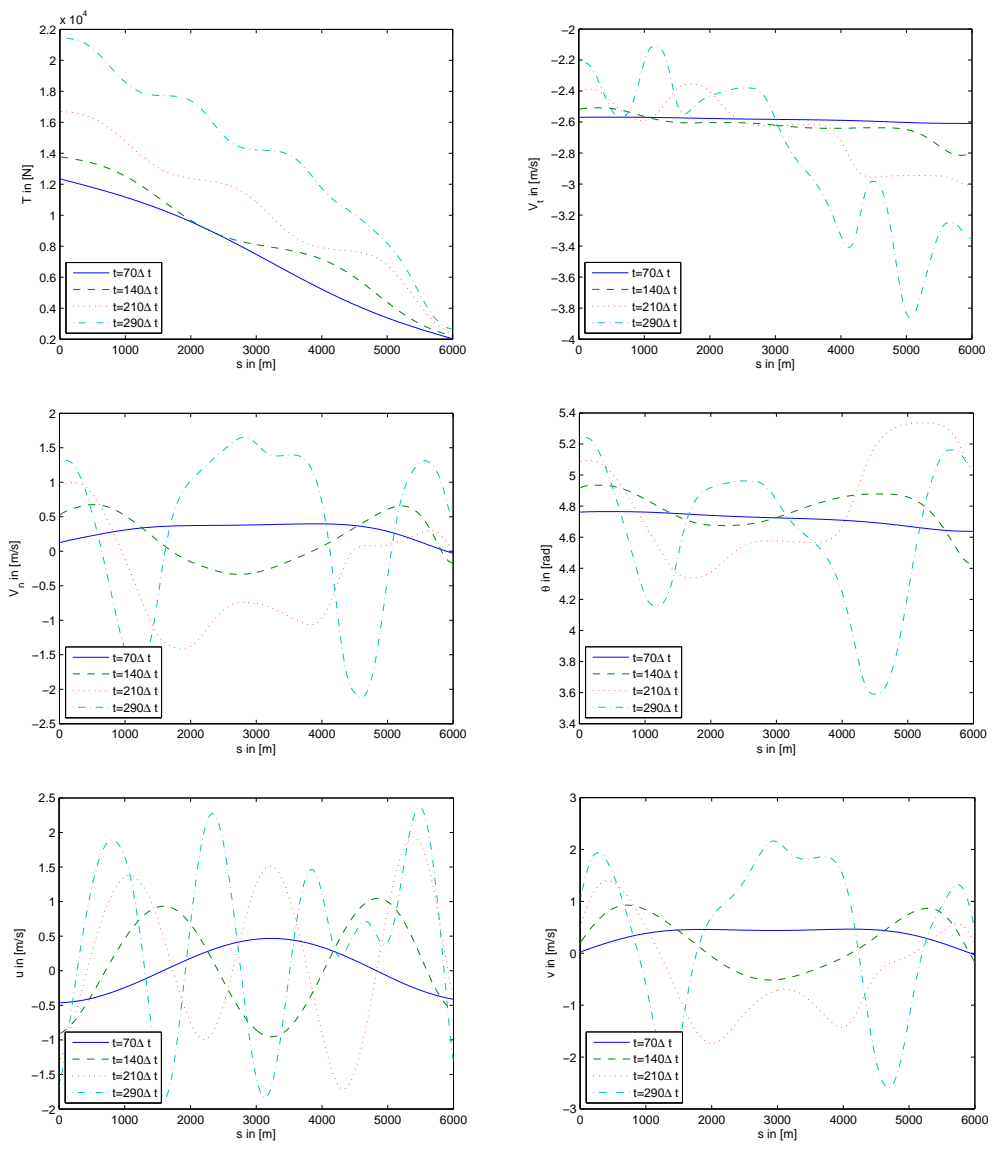

FiguRE 2. Forward solutions under smoothly varying inline (tangential) and cross-line (normal) hydrodynamic loads as defined in (15). From top left: the spatial profiles of tension, tangential velocity, normal velocity, and orientation angle along the cable of length $L=6000 \mathrm{~m}$. The bottom figures illustrate the ocean velocity profiles in the local coordinate frame. The plots refer to times $t=70,140,210$ and $290 \Delta t$ after the start of the motion, while the vessel executes a straight orbit with constant velocity of $2.57 \mathrm{~m} / \mathrm{s}$.

\section{Formulation OF THE INVERSE PROBLEM}

In the preceding sections we have examined the forward hydrodynamic model through the system of equations (1)-(9) in order to establish how the measurements relate to the ocean current velocities in a towed array survey system. Thus the forward model asserts the cable's shape and tension are affected by the hydrodynamic

disturbances. With an appropriate choice of boundary conditions the response of the system is uniquely determined (in the infinite dimensional sense), and subject to the discretization employed the uniqueness of the finite difference solution is granted when the Jacobian matrix in (14) has full rank. At an arbitrary point in 
time, consider the finite dimensional forward injective matrix-operator $\mathcal{B}$

$$
\mathcal{B}(u, v)=\mathbf{z}+\mathbf{w}
$$

mapping the ocean currents to the measurements within a tolerance set by the amount of instrumentation and physical noise expressed in the form $\mathbf{w}=\mathcal{N}\left(0, \sigma^{2}\right)^{1}$. In this context, the inverse problem seeks to infer the ocean currents from the knowledge of a finite set of noise infused measurements $\widetilde{\mathbf{z}}=\mathbf{z}+\mathbf{w}$. The reconstruction of $u$ and $v$ from (16) relies predominantly on the invertibility of the forward operator and the noise levels distorting the data. The differential form of the model equations (1) and (2) asserts that $\mathcal{B}$ is a quasi-linear integral operator that determines an unknown discrete function given its derivative and a boundary condition ${ }^{2}$. In effect, $\mathcal{B}^{-1}$ is a linear differential operator that maps a function to its first spatial derivative, and as such this is inherently rank deficient by one. This implies that in the knowledge of a function $f$ with $n$ degrees of freedom, its derivative $\mathcal{B}^{-1} f$ can only be uniquely specified with $n-1$ degrees of freedom, typically at the midpoints of the intervals of the $n$ samples. Consequently, the inverse problem of reconstructing the currents with $n$ degrees of spatial resolution given $n$ measurements is therefore rank-deficient ill-posed with immediate effects on the uniqueness and stability of the solution. For a review on the characteristics of linear rank deficient inverse problems we refer the reader to the textbooks (Hansen 1998) and (Vogel 2002). For the scope of this work it suffices to estimate the OCVP with $n-1$ degrees of freedom, which constitutes a somewhat 'milder' ill-posed problem since although the non-uniqueness is eradicated the stability concerns remain due to the impact of noisy data in conjunction with the differential forms of the model. The differentiation of noisy data is notoriously unstable and has long being the subject of research in signal processing (Wahba 1975), (Lu et al. 2006). To overcome the instability regularization is applied in order to construct a robust approximation of the operator $\mathcal{B}^{-1}$ using a priori information on the required ocean currents.

To formulate the regularized inverse problem we focus attention in the two momentum equations (1), (2) now expressed in terms of the noisy data quoted in tilde

$$
\begin{gathered}
\frac{\partial \widetilde{T}}{\partial s}=f_{t}(u)=\frac{1}{2} \rho \pi d C_{t}\left(\widetilde{V}_{t}-u\right)\left|\left(\widetilde{V}_{t}-u\right)\right| \\
\widetilde{T} \frac{\partial \widetilde{\theta}}{\partial s}=f_{n}(v)=\frac{1}{2} \rho d C_{n}\left(\widetilde{V_{n}}-v\right)\left|\left(\widetilde{V_{n}}-v\right)\right|
\end{gathered}
$$

For the noise content in the measured tension and curvature the derivatives on the left hand sides of (17) and (18) attain norms that grow arbitrarily large as $\Delta s \rightarrow 0^{3}$ (Lu et al. 2006), and thus in order to enforce stability we recast the equations using the integral 'anti-differentiation' operator

$$
(\mathcal{B} f)(s)=\int_{0}^{L} \mathrm{~d} s^{\prime} \mathrm{H}\left(s-s^{\prime}\right) f\left(s^{\prime}\right)
$$

\footnotetext{
${ }^{1}$ To describe the noise signal we use the normal distribution notation $\mathcal{N}\left(0, \sigma^{2}\right)$ indicating a Gaussian signal of zero mean and standard deviation $\sigma$.

${ }^{2}$ Here we have four measurement functions thus four boundary conditions (6)-(9) are needed for uniqueness. A more general case is treated in (Gatti 2002).

${ }^{3}$ Alternatively, to aid stability in computing the finite differences on the noisy data one would naively opt for increasingly larger discretization, fact which illustrates the intrinsic trade off between spatial resolution and stability in ill-posed problems.
} 
whose kernel is the Heavyside step function (Vogel 2002). On a finite grid with $n$ nodes the operator takes a matrix form of $\mathcal{B} \in \mathbb{R}^{n \times n-1}$ leading to integral formulations of the momentum equations like

$$
\underline{T}=\mathcal{B} f_{t}(u)
$$

and

$$
\underline{\theta}=\mathcal{B}\left(f_{n}(v) \oslash \widetilde{T_{a}}\right)
$$

where $\underline{T}=\widetilde{T}(L, t)-\widetilde{T}, \underline{\theta}=\widetilde{\theta}(L, t)-\widetilde{\theta}$ and ' $\oslash$ ' denotes element-wise division of equally sized vectors. In the same equations, $f_{t}(u), f_{n}(v)$ and $\widetilde{T_{a}}$ denote the element-wise average hydrodynamic loads and tension. Also note that the right hand side of (21) is always finite since the tension is bounded well above zero through condition (8). In order to obtain the tangential and normal drag forces from (20) and (21) respectively, we employ a generalized Tikhonov regularization scheme (Hansen 1998). As the required currents appear in distinct equations, these can be individually treated for regularization and solved separately. Applying Tikhonov regularization on (20) one seeks a solution $f_{t}^{*}(u)$ that minimizes the lease squares misfit in the measurements while satisfying also the smoothness constraint $\left\|\mathbf{D} f_{t}(u)\right\|_{2}^{2} \leq \kappa$ for a small scalar $\kappa$,

$$
f_{t}^{*}(u)=\arg \min _{\left\|\mathbf{D} f_{t}(u)\right\|_{2}^{2} \leq \kappa}\left\|\underline{T}-\mathcal{B} f_{t}(u)\right\|_{2}^{2}
$$

where $\mathbf{D} \in \mathbb{R}^{n-2 \times n-1}$ is a discrete form of the first-order difference operator, weighted so that $\|\mathbf{D}\|_{F} \sim\|\mathcal{B}\|_{F}{ }^{4}$. For a nonzero parameter $\lambda$, Tikhonov's formulation admits a unique analytic solution

$$
f_{t}^{*}(u, \lambda)=\left(\mathcal{B}^{T} \mathcal{B}+\lambda \mathbf{D}^{T} \mathbf{D}\right)^{-1} \mathcal{B}^{T} \underline{T}
$$

From the definition of the tangential hydrodynamic drag force the inline current distribution on the cable can be computed as

$$
u(\lambda)=\widetilde{V_{t a}}-\operatorname{sgn}\left(f_{t}^{*}(u, \lambda)\right) \odot\left(\frac{2\left|f_{t}^{*}(u, \lambda)\right|}{\rho \pi d C_{t}}\right)^{1 / 2}
$$

Following a similar procedure for (21) a Tikhonov regularized solution for the normal forces is obtained by

$$
f_{n}^{*}(v, \lambda)=\widetilde{T_{a}} \odot\left(\mathcal{B}^{T} \mathcal{B}+\lambda \mathbf{D}^{T} \mathbf{D}\right)^{-1} \mathcal{B}^{T} \underline{\theta}
$$

where $\odot$ denotes element-wise multiplication, and the cross-line currents are subsequently computed from

$$
v(\lambda)=\widetilde{V_{n a}}-\operatorname{sgn}\left(f_{n}^{*}(v, \lambda)\right) \odot\left(\frac{2\left|f_{n}^{*}(v, \lambda)\right|}{\rho d C_{n}}\right)^{1 / 2}
$$

where $\widetilde{V_{t a}}$ and $\widetilde{V_{n a}}$ are cable's element-wise average inline and cross-line velocities respectively.

\footnotetext{
${ }^{4}$ For a matrix $\mathbf{D} \in \mathbb{R}^{m \times n}$ with components $d_{i j}$ the Frobenius norm is $\|\mathbf{D}\|_{F}=$ $\sqrt{\sum_{i=1}^{m} \sum_{j=1}^{n}\left|d_{i j}\right|^{2}}$
} 


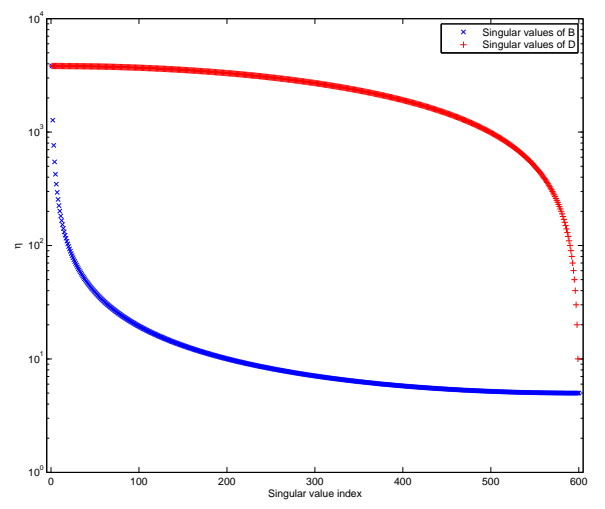

FiguRE 3 . The $n-1$ singular values of the integral operator $\mathcal{B}$ and the $n-2$ singular values of the regularization difference operator D on a logarithmic scale.

\section{Singular Value AND Sensitivity analysis}

In the formulation of the inverse problem the operators $\mathcal{B}$ and $\mathbf{D}$ as well as the regularization factor $\lambda$ have an important role. These are closely related in the sense that the difference operator $\mathbf{D}$ inverts the integral operator $\mathcal{B}$ and the regularization parameter scales their contribution in reconstructing the solution. Detailed analysis on this issue appears in many textbooks on inverse problem theory (Hansen 1998), (Vogel 2002) and will not by repeated here. For the scope of this work it suffices to demonstrate the relation of the two operators in terms of their singular value spectrum so that to aid an appropriate selection of $\lambda$. The graphs in figure 3 show the singular values of the operators discretized on a grid with 601 equally spaced nodes spanning over a $6000 \mathrm{~m}$ long cable. The inverse reciprocity between the exponentially decaying singular values of $\mathcal{B}$ and the logarithmically decreasing singular values of $\mathbf{D}$ reaffirms that $\mathcal{B} \mathbf{D}^{T}=I \in \mathbb{R}^{n \times n-2}$.

The singular value plots provide an insight into the problem of selecting the optimal regularization parameter $\lambda^{*}$. This parameter provides the necessary balance between prior and measurement-extracted information, by essentially weighting the biasing of the prior constraint to the objective of the minimization in (22). Consequently, this choice must be consistent with the noise levels in the measurements, the uncertainty in the model parameters and the credibility of the available prior information. The optimal parameter is known to satisfy $\min (\eta)<\sqrt{\lambda}<\max (\eta)$ where $\eta_{1}, \ldots, \eta_{n-1}$ are the singular values of $\mathcal{B}$, and it is often computed using the L-curve (Hansen 1998) or the generalized cross validation method (Wahba 1975).

While some of the model parameters like the cable's length and diameter can be measured with great accuracy, others are somewhat difficult to coin exactly. The drag coefficients $C_{t}$ and $C_{n}$ for example are assigned values after a series of laboratory controlled experiments, and although it may seem reasonable to assume that these are known with a small level of uncertainty, one should examine in some rigor the impact of such uncertainties to the performance of the inverse method. 
From the model equations it is clear that errors in $C_{t}$ affect merely the computation of the inline current and those in $C_{n}$ that of the cross-line velocity component. If the tangential coefficient is known exactly then in assuming noise free measurements the exact inline current $u^{*}$ is obtained from (24) for a given small value of $\lambda$. Introducing a small perturbation $\delta$ so that $C_{t} \rightarrow C_{t}+\delta$ can be shown to yield a profile $u$ with a square error

$$
\epsilon_{\delta}^{2}(u)=\left(u^{*}-u\right)^{2}=\frac{2}{\rho \pi d}\left|\frac{\partial T}{\partial s}\right|\left[\sqrt{\frac{1}{C_{t}}}-\sqrt{\frac{1}{C_{t}+\delta}}\right]^{2}
$$

where we have used the fact that the gradient of the tension is always negative. To aid comparison we now apply the same perturbation to the exact normal drag coefficient like $C_{n} \rightarrow C_{n}+\delta$ and compute the square error on the cross-line velocity as

$$
\epsilon_{\delta}^{2}(v)=\left(v^{*}-v\right)^{2}=\frac{2}{\rho d}\left|T \odot \frac{\partial \theta}{\partial s}\right|\left[\sqrt{\frac{1}{C_{n}}}-\sqrt{\frac{1}{C_{n}+\delta}}\right]^{2}
$$

where $v^{*}$ the exact solution obtained from (26). As the nominal values of the drag coefficients satisfy $C_{t} \ll C_{n}$ it is clear that $\left[\sqrt{C_{t}^{-1}}-\sqrt{\left(C_{t}+\delta\right)^{-1}}\right]^{2} \gg\left[\sqrt{C_{n}^{-1}}-\right.$ ${\sqrt{\left(C_{n}+\delta\right)^{-1}}}^{2}$ but this does not necessarily imply that $\epsilon_{\delta}(u) \gg \epsilon_{\delta}(v)$ for the same $\delta$. In fact the influence of the tension in the formula for $\epsilon_{\delta}(v)$ is critical and although this relates inversely proportional to the curvature, in the range of angles relevant to the application the factor $T \odot \partial \theta / \partial s$ can become arbitrarily large, especially toward the tow-point. Moreover, as it has been reported in (Grosenbaugh 1991), flow-induced vibrations occurring during unsteady towing motion may cause the cable's drag coefficients to vary substantially from their nominal values. In particular, it is possible that the normal coefficient alters, heterogeneously on $s$, by as much as $40 \%$ during the motion. In such a case the large offset on $C_{n}$ is likely to yield misleading results for the reconstructed cross-line currents. To avoid this the proposed inverse methodology needs to be reformulated in the context of robust parameter estimation (Ben-Tal et al. 1998) in order to allow for a degree of uncertainty on the distribution of the drag coefficients along the cable.

In order to demonstrate the robustness and resolution of the inverse method in reconstructing velocity profiles where one of the two components is dominant some additional simulations have been performed. In the first case we assume large homogenous velocities $c_{x}(x, t)$ normal to the originally straight cable configuration and low magnitude smoothly varying currents $c_{y}(y, t)$ oriented parallel to the initial trajectory. Assuming towing at constant speed of $2.57 \mathrm{~m} / \mathrm{s}$ on a northbound straight orbit, we set $c_{x}(x, t)=10 \mathrm{~m} / \mathrm{s}$ and $\max \left|c_{y}(y, t)\right|=2 \mathrm{~m} / \mathrm{s}$. The resulted normal drag force causes the cable to gradually deviate from the vessel trajectory eventually attaining a zero curvature configuration at a large angle $\theta$. As the graphs at the top row of figure 4 suggest, the impact of high normal velocities becomes more profound toward the low-tension end of the cable, while this adjusts its shape in order to minimize the effect of normal currents. As $\theta$ increases the graphs show that the cable nearly straightens, despite a small curvature due to the $c_{y}$ currents, so that eventually the dominant $c_{x}$ is largely projected onto the $\mathbf{t}$ axis. In the reverse scenario where the currents are predominantly parallel to the cable the tension rises and because of the boundary condition (8) the absolute value of the gradient of the tension increases and this maintains the curvature at low levels. 

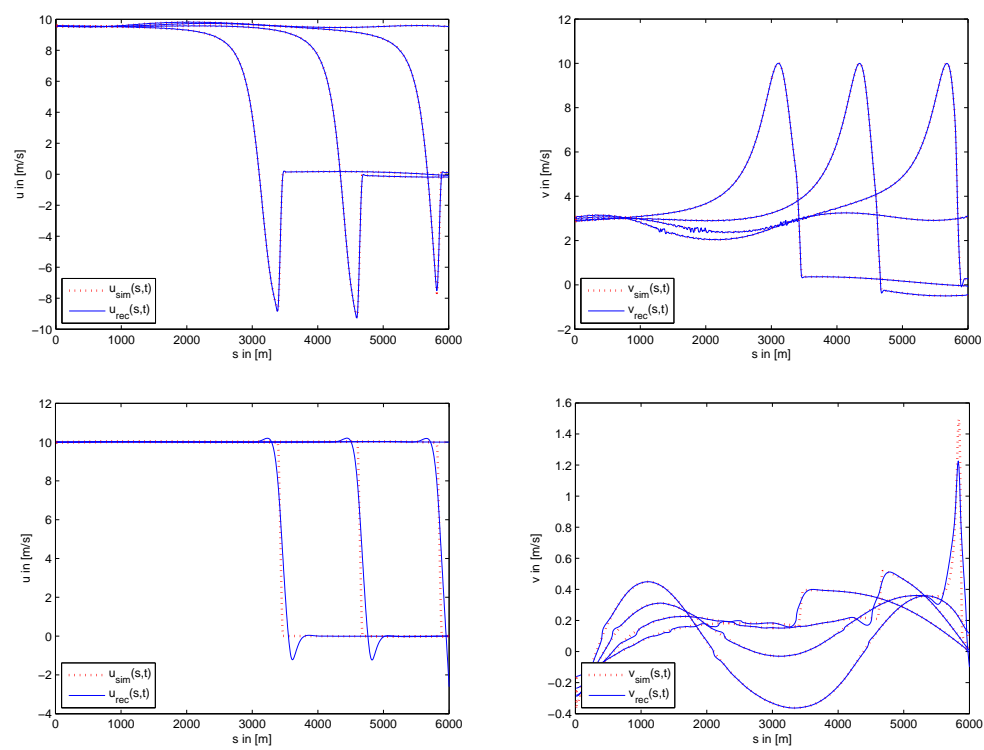

FiguRE 4. Simulated and reconstructed ocean velocities at times $60,80,100$, and $120 \Delta t$ from the beginning of motion. Top row dominant $c_{x}$ currents and bottom row dominant $c_{y}$ currents.

The results appearing at the bottom row of figure (4) have been computed with $c_{y}(y, t)=-10 \mathrm{~m} / \mathrm{s}$ and $\max \left|c_{x}(x, t)\right|=2 \mathrm{~m} / \mathrm{s}$ at a same towing speed and orbit. In such a situation the accuracy of the positioning data that determines the shape and the curvature becomes critical, since the sensitivity of the method to in-line currents relates quasi-linearly to the product of the tension to changes in curvature, hence deviations from the straight trajectory will imply higher normal currents. The reconstruction formulae (23) and (25) clearly indicate that the sensitivity of the method to inline currents relies exclusively on resolving the gradient of the tension profile along the cable. In this regard, cables with high Young modulus will have improved sensitivity in detecting tangential currents. On the other hand, the detection of cross-line currents is manifested by the observed changes in the cable's shape and curvature with in turn relates to its flexibility. Consequently, cables with low modulus of rigidity ar expected to have a higher sensitivity in normal currents.

\section{REsults AND DisCUSSION}

The inverse problem under consideration is a special case of the classical problem of numerical differentiation of noisy data in the context of regularization ( $\mathrm{Lu}$ et al. 2006). In this section we provide simulated benchmark examples which aim to test the noise robustness of the inverse method. In this we illustrate the impact of noise on the ill-posed inverse problem and subsequently demonstrate how this can be eliminated using Tikhonov regularization. Emphasis is also drawn in the calibration of the regularization parameter for the various noise levels. The simulation conditions used are similar to those discussed in section 5 from which discrete sets of tension and positioning measurements have been simulated at time 

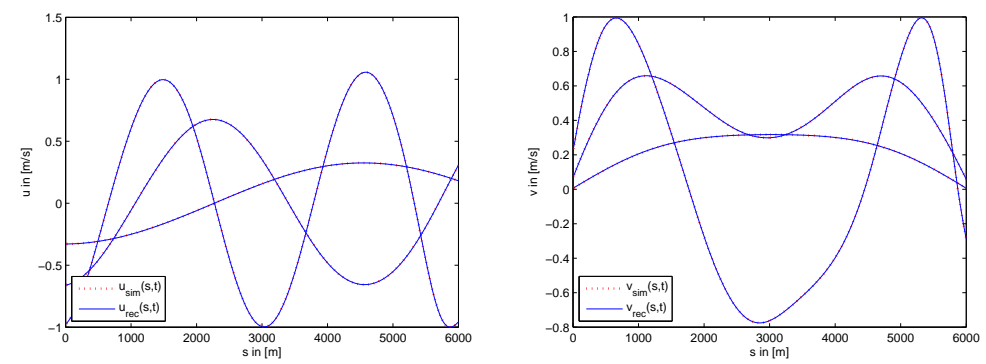

Figure 5. Noise free results. At left the simulated and reconstructed tangential (inline) OCVP at $t=50,100,150 \Delta t$ after the start of the simulation, and right the corresponding normal (crossline) OCVP.

intervals $\Delta t=10 \mathrm{~s}$. For the solution of the inverse problem a coarser grid with 600 elements of $\Delta s=10 \mathrm{~m}$ has been employed.

6.1. Reconstructing noise-free data. In the first instance we consider the ideal case where one possesses noise free measurements $\widetilde{\mathbf{z}}=\mathbf{z}$, from which the OCVP can be recovered directly from the model equations. This can be achieved either by evaluating the gradients in (17) and (18) using a finite difference scheme, or via the integral equations (23) and (25) for zero amount of regularization. Opting for the latter choice and setting $\lambda=0$, we obtain at time instants $t=50,100$ and $150 \Delta t$ the current profiles appearing in figure 5 . The graphs illustrate an exact match between simulated and reconstructed profiles, This is coherent with the analysis on the properties of the integral operator made in section 5 . In fact for zero regularization $\mathcal{B}^{-1}$ takes the form of a Moore-Penrose pseudo-inverse that has $n-1$ nonzero singular values. The noise-free example serves as a measure of comparison for the performance of the inverse method under realistic noise levels explored next.

6.2. Reconstructing noisy data. The necessity for regularization becomes more profound when data are infused with additive random noise. This is typically the situation arising in the acquisition of real survey data where these are corrupted by instrumentation errors and physical noise. To emulate these conditions we introduce a pseudo-random white noise signal $\mathbf{w}=\mathcal{N}\left(0, \sigma_{1}^{2}\right)$ on the forward variables where $\sigma_{1}=10^{-2} \bar{z}$, and $\bar{z}$ denotes the mean value of $T, V_{t}, V_{n}$ and $\theta$. The simulated OCVP at times $t=300,400 \Delta t$ appear in the graphs of figure 6 along with the reconstructed profiles for various values of the regularization parameter. Each graph illustrates the reconstructed solution obtained by the Tikhonov method using (24) and (26) for $\lambda=0.1,10$ and 100 in order to demonstrate the impact of the parameter on the spatial resolution of the reconstructed velocities. In adopted generalized Tikhonov scheme with a priori smoothness assumptions as imposed by the selection of $\mathbf{D}$ operator, $\lambda$ controls the degree of smoothness in the inverse solution. If $\lambda^{*}$ is the optimal value of the parameter according to the noise content in the data, the graphs show that using a $\lambda \ll \lambda^{*}$ under-regularizes the inverse problem, and thus the effect of noise is profound. On the other hand, a choice $\lambda \gg \lambda^{*}$ over-regularizes the problem, effectively filtering some detailed features of the solution and hence 

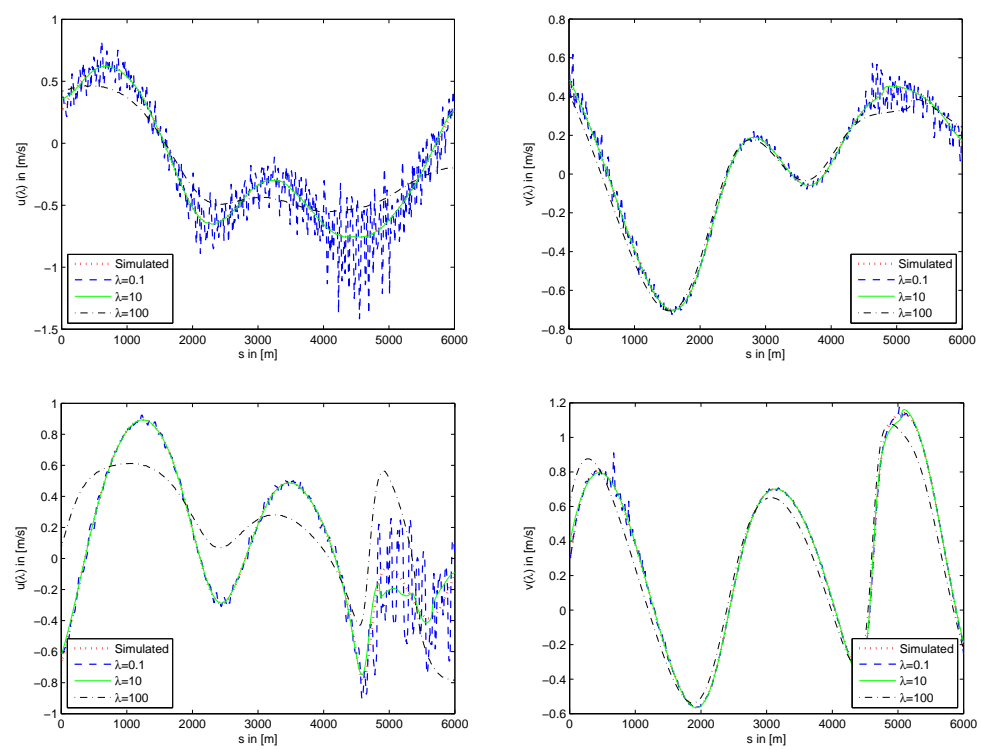

FiguRE 6. Reconstructed OCVP with Gaussian noise of zero mean and standard deviation $\sigma_{1}$. At the top the simulated and reconstructed OCVP at $t=300 \Delta t$ after the start of the simulation. The three inverse solutions have been obtained using Tikhonov regularization for three different values of the regularization parameter $\lambda$. The plots are indicative of under-regularization (dashed), optimal regularization (solid) and over-regularization (dash-dotted). At the bottom the simulated and reconstructed OCVP with the same values of $\lambda$ at $t=400 \Delta t$.

compromising the spatial resolution. The optimal value of $\lambda^{*} \approx 10$ was computed using the L-curve method (Hansen 1998).

Observing the graphs of the same figure there is another interesting remark to be made. It appears that the reconstruction of the normal component $v$ is qualitatively and quantitatively superior compared to that of $u$ for all values of the regularization parameter. This is in fact justified since, despite their evocative formulation similarity, inversion formula (25) offers significantly better noise robustness compared to $(23)$, since the tension averaging in the form of the vector $\widetilde{T_{a}}$ which multiplies the regularized inverse of the curvature in the former equation, eradicates most of the noise in the contaminated tension data, and since by default $\|\widetilde{T}\|_{2} \gg\|\widetilde{\theta}\|_{2}$ (c.f. figure 2), the impact of noise on (25) is limited.

As a second case we consider simulated data under more intense noise conditions, captured at times $t=500 \Delta t$ and $t=600 \Delta t$ after the start of motion. In the scenario the towing vessel was set to execute a circular orbit with a radius of $3000 \mathrm{~m}$ at the same constant speed of $2.57 \mathrm{~m} / \mathrm{s}$. The noise signal infused with the measurements was set to $\mathbf{w}=\mathcal{N}\left(0, \sigma_{2}^{2}\right)$ for $\sigma_{2}=3 \times 10^{-2} \bar{z}$. As before, we proceed to compute the regularized hydrodynamic loads and velocities along the cable by estimating first the optimal regularization parameter. As anticipated, with the increase in the level of noise in the data, a rise in the amount of smoothing is required to stabilize 

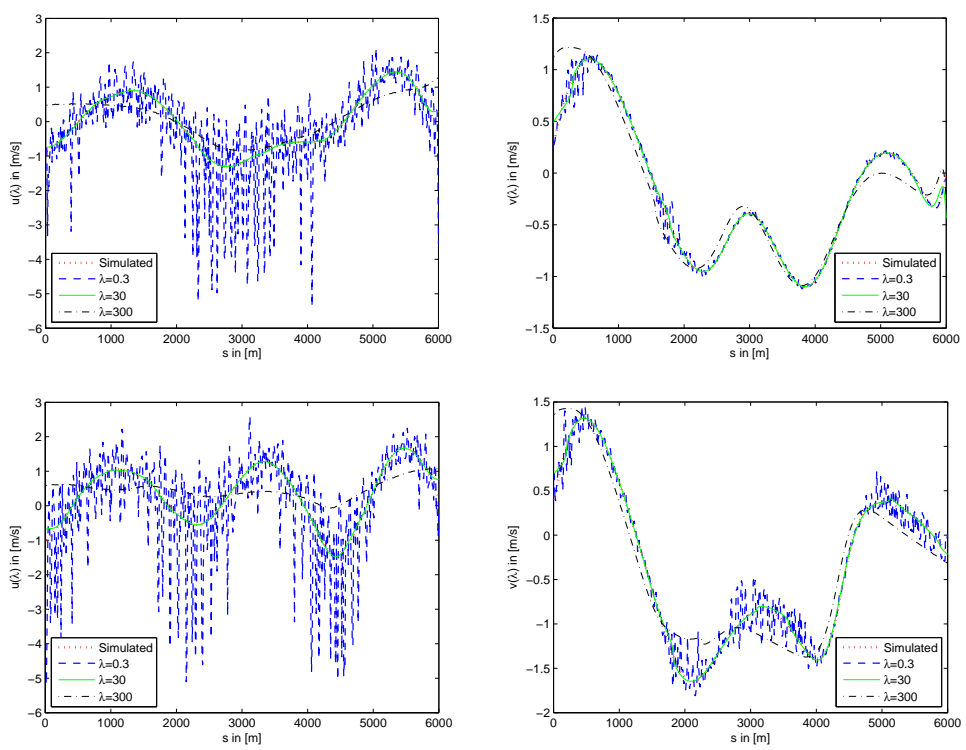

FiguRE 7. Reconstructed OCVP with Gaussian noise of zero mean and standard deviation $\sigma_{2}=3 \sigma_{1}$. At the top the simulated and reconstructed currents at $t=500 \Delta t$ after the start of the simulation. The three inverse solutions have been obtained using Tikhonov regularization for three different values of the regularization parameter $\lambda$. The plots are indicative of under-regularization (dashed), optimal regularization (solid) and over-regularization (dash-dotted). Below, the corresponding currents with the same values of $\lambda$ at $t=600 \Delta t$.

the solution. The implementation of L-curve method yielded an optimal value for the parameter around $\lambda^{*} \approx 30$. The simulated and reconstructed velocity profiles at times $t=500 \Delta t$ and $t=600 \Delta t$ are presented in figure 7 . Compared to the results of the previous case, the spatial resolution of the optimally reconstructed solutions $u\left(\lambda^{*}\right)$ and $v\left(\lambda^{*}\right)$ is somewhat compromised by the noise distortion in the measurements, while as before the reconstruction of the tangential component is affected the most. In the same graphs we provide also some inverse solutions obtained with non-optimal regularization in order to illustrate the influence of the parameter on the solution. In particular, for a $\lambda<1$ the amount of the filtering imposed on the spectrum of $\mathcal{B}$ does not suffice to stabilize the solution and the method fails to yield an informative solution.

6.3. Angle of attack prediction. One of the fundamental aspects of towed array seismic imaging, directly related to the optimal steering of the cable, is the estimation or prediction of the angle of attack. As described in the preceding sections, the reconstruction of the OCVP provides some significant input to the systems that control the steering of the cable, and in this sense the angle of attack embodies this information. More precisely the knowledge of the ocean velocities impinging on the 

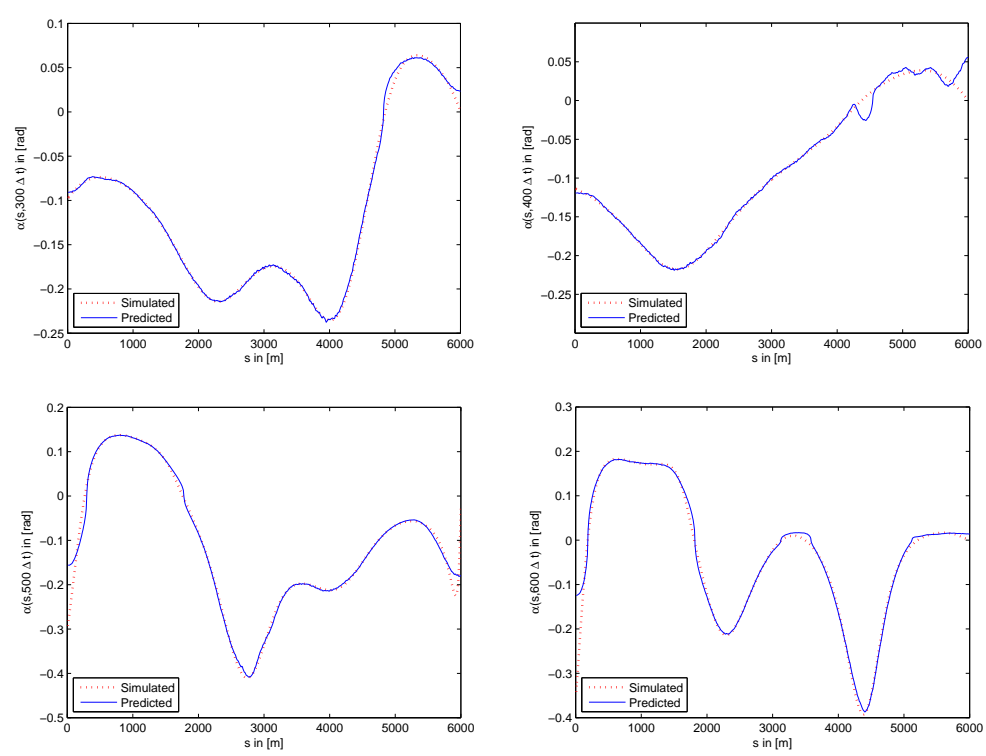

FigURE 8. Angle of attack prediction performance. The simulated versus predicted angle of attack at $t=300,400,500,600 \Delta t$ after the start of the simulation, obtained with the second set of noisy data.

streamer can yield an estimate on the angle of attack $\alpha$,

$$
\alpha=\alpha_{c}+\arctan \left(\frac{V_{n}-v}{V_{t}-u}\right)
$$

so that the feedback control angle $\alpha_{c}$ can be adjusted in a way that the cable is steered to the desired location irrespectively of the ocean currents. In figure 8 we plot the graphs of the simulated and predicted profiles for the angles of attack at times $t=300,400,500,600 \Delta t$ for the data of the second simulation above under optimal regularization. The results show a good agreement between simulated and predicted $\alpha$ profiles under noise conditions, fact which justifies the utility of the inverse method in assisting the optimal steering of the streamer.

\section{Conclusions}

In this paper we present a complete framework for solving the forward and inverse hydrodynamic problems in towed array seismic survey. Our main contribution rests with the inverse problem of inferring the ocean current velocity profiles when the shape and tension variation along the cable are approximately known. In the context of regularization in inverse problem theory, we have presented a robust methodology for reconstructing the ocean velocities and predicting the corresponding angle of attack. Using simulated case studies we have demonstrated the noise robustness of the proposed method under the optimal regularization through the calibration of the involved parameter. Moreover, the results indicate that adequate 
spatial resolution is realistically feasible despite the noise content in the measurements when smooth velocity profiles are sought through the implementation of a generalized Tikhonov regularization in the inverse solver.

\section{ACKNOWLEDGEMENTS}

The authors would like to express their gratitude to Smith Institute for Industrial Mathematics for organizing the 'Study Group with Industry' that brought together the second and third authors to work together on this problem. The authors would also like to thank Dr J.O. Paulsen of WesternGeco at Oslo technology center for his help and support of this work. The second author is now with Validus Engineering AS.

\section{REFERENCES}

Ablow C.M. and Schechter S., Numerical simulation of undersea cable dynamics Ocean engineering, vol. 10, no. 6, pp 443-457, 1983.

Ben-Tal A. and Nemirovski A., Robust convex optimization, Mathematics of Operations Research, vol. 23, no.4, 1998.

Dowling A.P., The dynamics of towed flexible cylinders. Part 1: Neutrally buoyant elements, Journal of Fluid Mechanics, vol. 187, pp. 507-532, 1988.

Gatti C.S.C., Numerical simulations of large deformation cable dynamics, University of Michigan Ph.D. Thesis, 2002.

Gobat J.I., The dynamics of geometrically compliant mooring systems, MIT-WHOI Ph.D. Thesis, 2000.

Gobat J.I and Grosenbaugh M.A., Application of the generalized $\alpha$ method to the time integration of the cable dynamics equations, Computer Methods in Applied Mechanics and Engineering, vol. 190, pp 4817-4819, 2001.

Grosenbaugh M.A., The effect of unsteady motion on the drag forces and flowinduced vibrations of a long vertical tow cable, International Journal of Offshore and Polar Engineering, vol. 1, no. 1, 18-26, 1991.

Hansen P.C., Rank deficient and discrete ill-posed problems: Numerical aspects of linear inversion, SIAM, Philadelphia, 1998.

Hover F.S., Grosenbaugh M.A and Triantafyllou M.S., Calculation of dynamic motions and tensions in towed underwater cables; IEEE Journal of Oceanic Engineering, vol. 19, no. 3, 1994.

Hui Z., Measuring ocean current from a towed seismic array at real time; M.Sc. Thesis, UMIST, 2005.

Hughes T.J.R., The finite element method: Linear static and dynamic finite element analysis; Prentice-Hall, New Jersey, 1987.

Lu S. and Pereverzev V., Numerical differentiation from the viewpoint of regularization theory, Mathematics of Computation, vol. 75, no. 256, 2006.

Matlab: The language of technical computing, The Mathworks Inc.

Milinazzo F., Wilkie M. and Latchman S.A., An efficient algorithm for simulating the dynamics of towed cable systems, Ocean Engineering, vol. 14, no. 6, pp 513$526,1987$.

Nakstad H., and Kringlebotn J.T., Probing oil fields, Nature Photonics, vol. 2, pp 147-149, 2008.

Storteig E. and Lionheart W., 2007, Current prediction in seismic surveys, US patent 20070127312 . 
Vogel C., Computational methods for inverse problems, SIAM, Philadelphia, 2002.

Wahba G., Smoothing noisy data with spline functions, Numerische Mathematik, vol. 24, no. 5, 1975.

†Mit Energy Initiative, Massachusetts Institute of Technology, USA

$\ddagger$ WesternGeco, Solbråveien 23, 1383 Asker, Norway

$\S$ School of Mathematics, University of Manchester, UK 